\title{
Surgical Technique: Treatment of Distal Humerus Nonunions
}

\author{
Johanna C. E. Donders, MD • Dean G. Lorich, MD • David L. Helfet, MD • Peter Kloen, MD, PhD
}

Received: 18 August 2016/Accepted: 2 March 2017/Published online: 12 April 2017

(C) The Author(s) 2017. This article is published with open access at Springerlink.com

\begin{abstract}
Background: Open reduction and internal fixation of distal humerus fractures is standard of care with good to excellent outcome for most patients. However, nonunions of the distal humerus still occur. These are severely disabling problems for the patient and a challenge for the treating physician. Fortunately, a combination of standard nonunion techniques with new plate designs and fixation methods allow even the most challenging distal humeral nonunion to be treated successfully. Questions/ Purposes: The purpose of this manuscript is to describe our current technique in treating distal humeral nonunion as it has evolved over the last four decades. We have now follow-up on 62 treated patients. Methods: A few key steps are essential to obtain bone healing while regaining or preserving elbow motion. These include careful planning, extensile exposure, release of the ulnar nerve, capsular release and mobilization of the distal fragment, debridement, and finally stable fixation after alignment with application of bone graft. Results: The vast majority of distal humeral nonunions can be treated successfully with open reduction and internal fixation. Conclusion: Important components of the treatment plan are careful preoperative planning, extensile
\end{abstract}

Work performed at Academic Medical Centre, Amsterdam, the Netherlands and the Hospital for Special Surgery, New York, NY.

Level of Evidence: Therapeutic Study Level IV

Electronic supplementary material The online version of this article (doi:10.1007/s11420-017-9551-y) contains supplementary material, which is available to authorized users.

J. C. E. Donders, MD • P. Kloen, MD, PhD ( $\varangle)$

Department of Orthopedic Surgery, Academic Medical Centre, University of Amsterdam,

Meibergdreef 9,

Amsterdam, 1105 AZ, The Netherlands

e-mail: p.kloen@amc.uva.nl

J. C. E. Donders, MD • D. G. Lorich, MD • D. L. Helfet, MD

Orthopaedic Trauma Service, Hospital for Special Surgery,

535 East 70th Street,

New York, NY 10021, USA

J. C. E. Donders, MD • D. G. Lorich, MD • D. L. Helfet, MD

Weill Cornell Medical College,

New York, NY 10065, USA approach, debridement, and solid fixation with—-locking — plates and liberal use of bone graft.

Keywords nonunion - distal humerus · bone graft · internal fixation

\section{Introduction}

Most often, a distal humerus nonunion is located at the supracondylar level with the articular fragments having healed in a near-anatomic position. Motion at the nonunion site causes pain, limited elbow function, and disability [1-4, 8-12, 21-23]. Hardware will ultimately fail or loosen with often a windshield wiper effect of the screws in the bone, further compromising bone stock $[9,10]$. The increased motion at the supracondylar level, excessive scar formation, and inflammation around the ulnar nerve can lead to nerve symptoms including pain, numbness, and/or paresthesias. Use of the arm for loaded activities and positioning the forearm and hand against gravity will be severely compromised.

A nonunion of the distal humerus is often oligotrophic, being a combination of decreased biological activity and insufficient stability. They can be sub-classified based on location being supracondylar, transcondylar, intercondylar, unicondylar (medial or lateral), or osteochondral $[13,19]$. The size of the distal fragment can be underestimated as it is often flexed on the APradiograph. A CT scan will help determine the actual size (Fig. 1) [10]. In addition, for complex cases, we nowadays print 3D models of the nonunion and compare it to the mirror-imaged healthy side to help planning (Fig. 2). It is always important to consider an associated infection, even if there are no clear symptoms as fever, drainage, or wound problems.

Treatment Options Elbow arthrodesis, distraction arthroplasty [14], allograft [24], open reduction and internal fixation [1-4, 8$12,16,19,20,22,23]$, prosthetic replacement $[6,7,12,15,17$, $18]$, and the Ilizarov technique $[5,20]$ have all been used in the management of distal humeral nonunions. Each of these treatment options has limitations and complications, including difficult bone fusion (due to restricted bone stock), inconsistent outcomes, instability, weakness and loss of function of the elbow joint, neuropathic joints, and infection. 


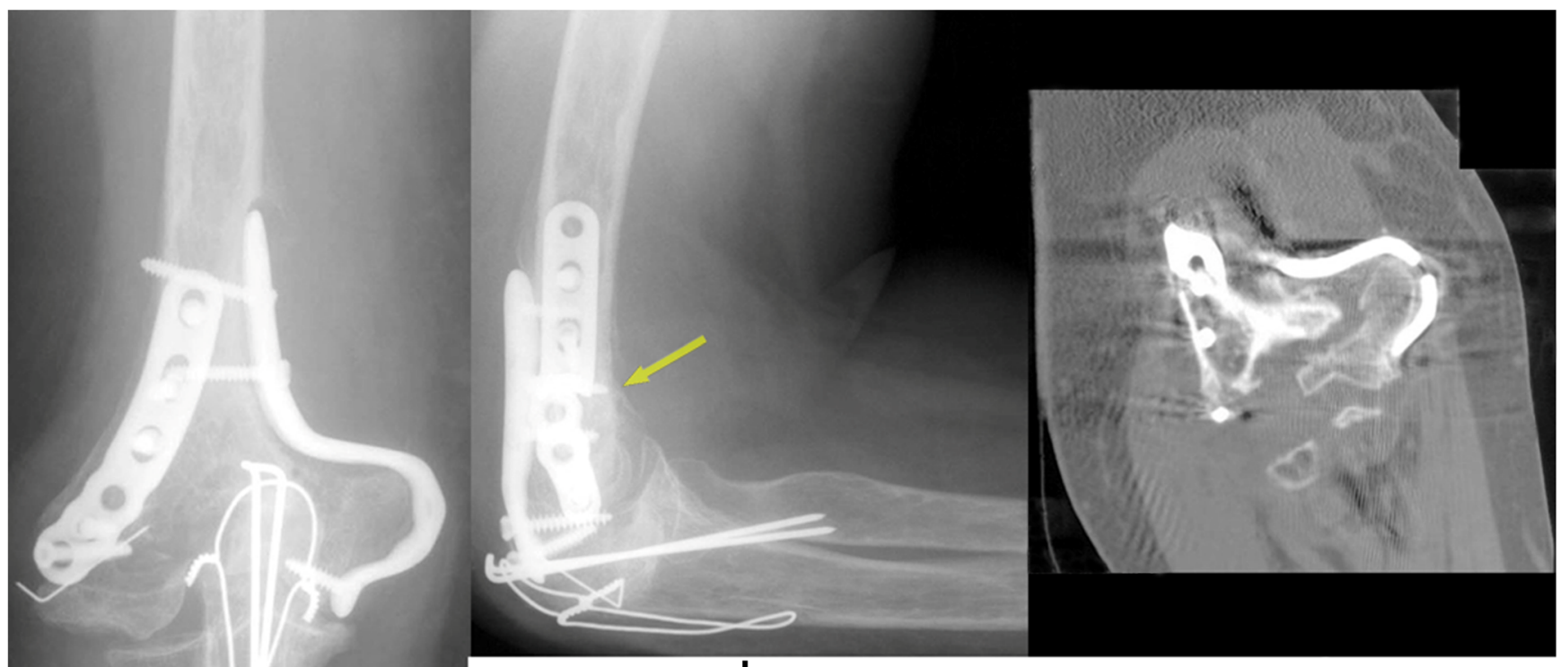

b

C

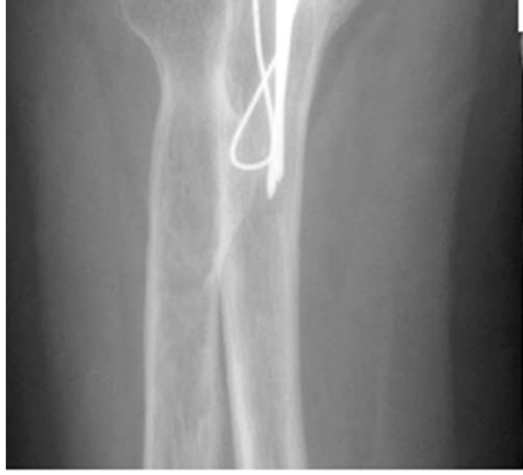

a

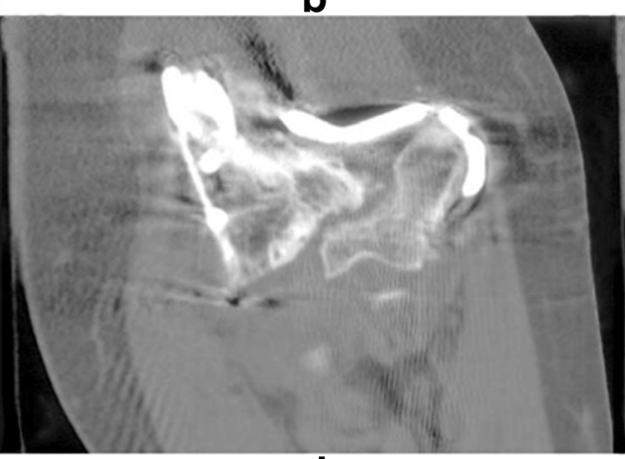

d

Fig. 1. A 60 -year-old female presents 8 months following ORIF of a right-sided distal humeral fracture with complaints of pain at the fracture site and limited range of motion of the elbow. Radiographs $(\mathbf{a}, \mathbf{b})$ and CT scan images $(\mathbf{c}-\mathbf{e})$ reveal a nonunion, and loss of fixation with plate breakage (medial side).

Our experience is that most nonunions of the distal humerus can be treated surgically to obtain bone healing with a functional range of motion of the elbow. We described in 2002 what, to the best of our knowledge, is the largest series of 52 distal humeral nonunions treated with internal fixation [9]. Since this publication, new anatomic locking plates have been introduced allowing better fixation of small osteopenic nonunion fragments. The aim of this manuscript is to describe our current treatment of a distal humeral nonunion using a standardized treatment plan that includes careful planning, extensile exposure, release of the ulnar nerve, capsular release and mobilization of the distal fragment, debridement, and finally stable fixation after alignment with application of bone graft. We also provide a summary of the pertinent literature.

\section{Methods}

\section{Surgical Technique}

\section{Preparation and Positioning}

The patient is positioned prone or in the lateral decubitus position. In the presence of an osteochondral shearing-type nonunion, a lateral approach might be better. A preoperative drawing outlining the surgical tactic will help anticipate and prevent intraoperative problems (Fig. 3). The patient is positioned supine with the arm abducted on an arm-table. No antibiotics are given until a minimum of five deep cultures are obtained.

\section{Exposure}

The posterior approach allows elevation of medial and lateral skin flaps and an olecranon osteotomy. The ulnar nerve is identified proximally and traced towards the elbow. If ulnar nerve symptoms are present preoperatively, external neurolysis is performed. The olecranon osteotomy for an intra-articular, transcondylar, or a low distal humeral nonunion provides superb exposure and ability for extensile release (Fig. 4). For a supracondylar distal humeral nonunion, a triceps splitting, paratricipital, or triceps-reflecting anconeus pedicle approach can be used. For a unicondylar nonunion, a medial or lateral approach is often sufficient. Alternatively, one can start with a paratricipital approach and add an olecranon osteotomy as needed. The chevron olecranon osteotomy is done at the semilunar notch where the cartilage is thinnest. Careful elevation of the proximal fragment prevents articular separation that can be the result of 

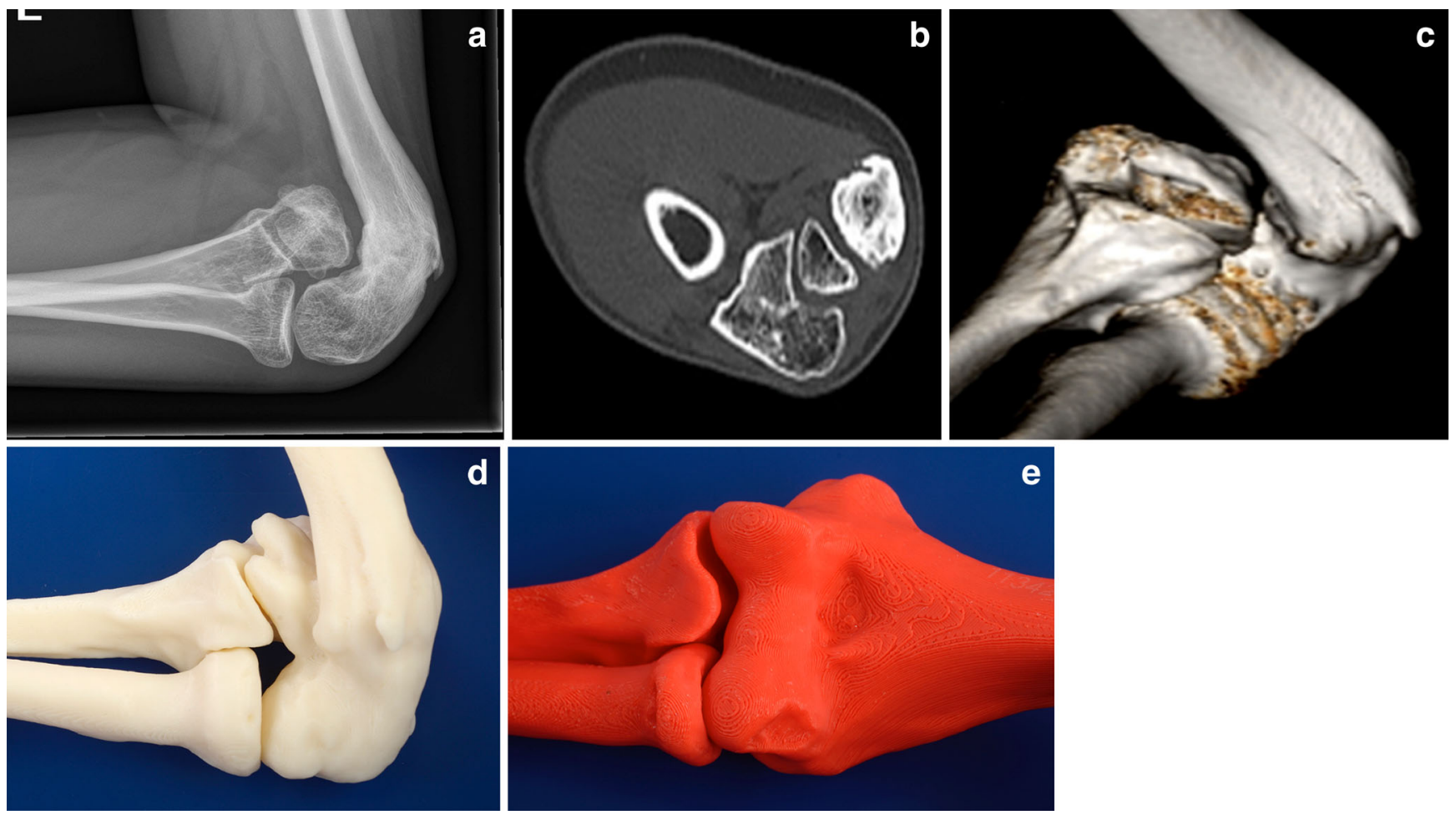

Fig. 2. For complex cases, a 3D CT and 3D models of the affected and the mirror-imaged healthy elbow will provide better insight in the nonunion and associated deformity. This patient had a war-related injury to his elbow as a child and presented 11 years later. Plain radiographs suggested an elbow dislocation as seen on the lateral radiograph (a). 2D CT imaging (b) and 3D-reformatted CT imaging (c) showed a malunion of the distal humerus with associated medial condyle nonunion. In addition, there was overgrowth of the radial head and capitellum. The proximal radio-ulnar joint, the radio-capitellar joint, and the relation between the proximal ulna and medial condyle nonunion are intact. 3D-printed models of the affected (d) and mirror-imaged healthy side (red) (e) provide valuable insight.
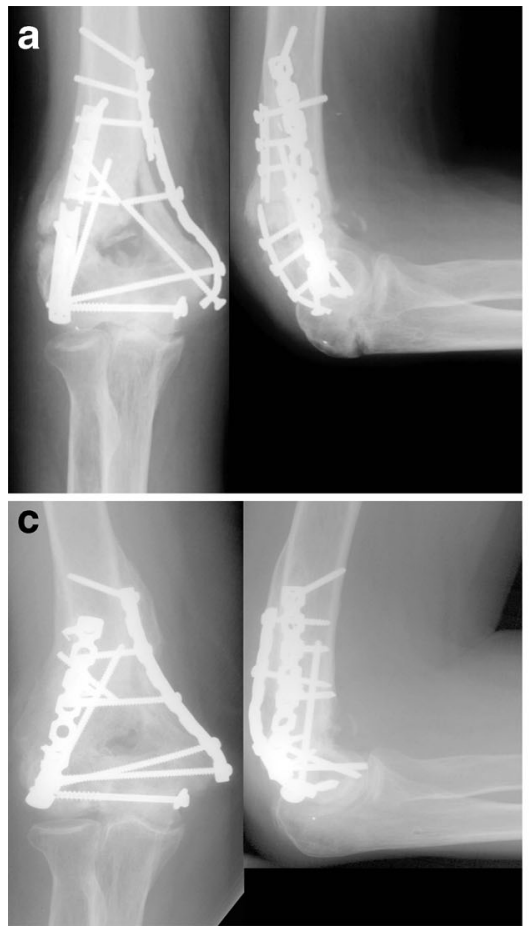

b

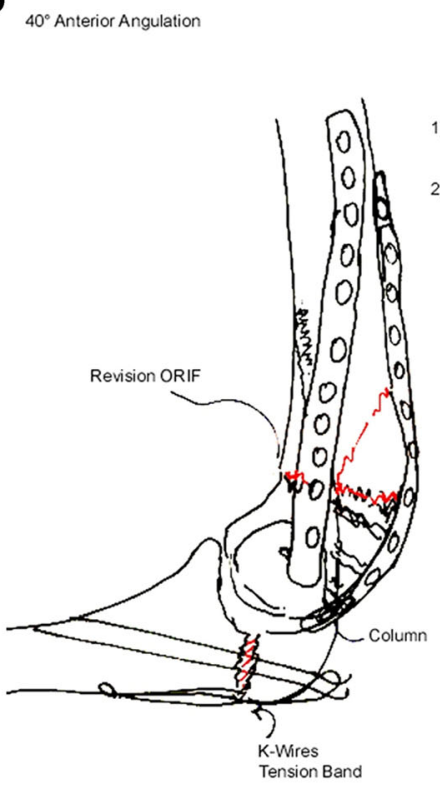

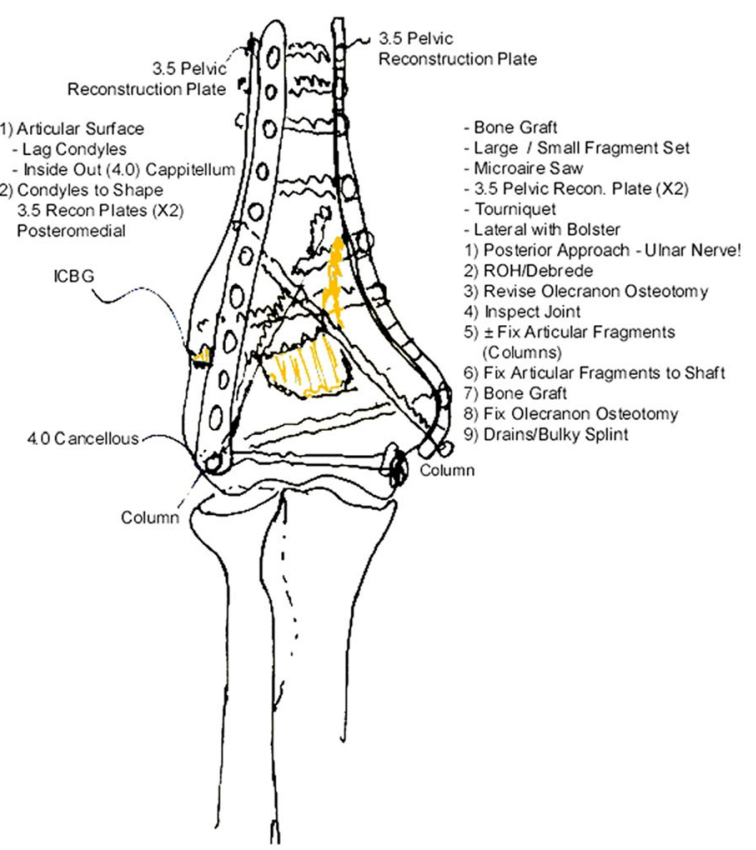

Fig. 3. Good preoperative planning will greatly facilitate the surgical procedure. Preoperative anteroposterior (AP) and lateral radiographs (from left to right) show a distal humerus nonunion with failed hardware (a), detailed pre-op plan (b), and final AP and lateral radiographs (from left to right) illustrating a healed distal humerus nonunion (c). 

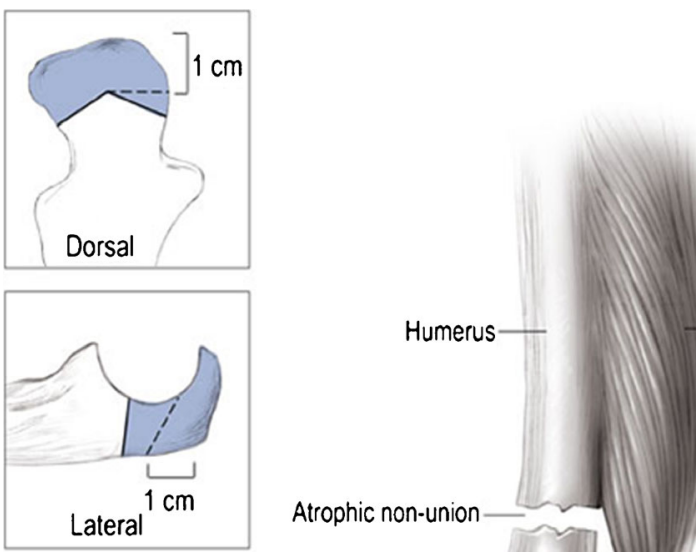

Atrophic non-union

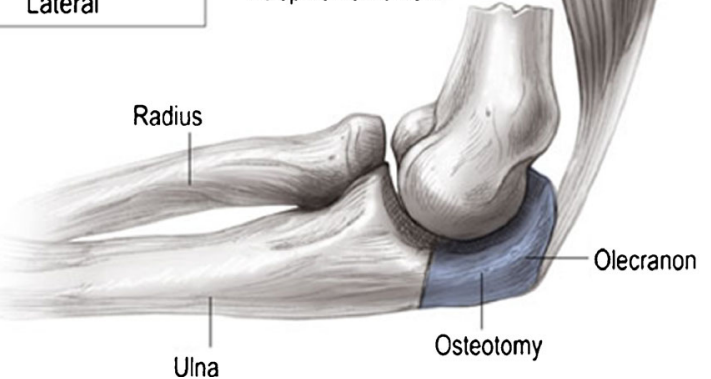

Fig. 4. The olecranon osteotomy is angulated (as shown in inset), forming an apex to facilitate reduction and providing additional rotational stability for fixation (from: Helfet DL, Kloen P, Anand N, Rosen HS. ORIF of delayed unions and nonunions of distal humerus fractures. Surgical technique. J Bone Joint Surg Am. 2004;suppl 1:18-29. Reprinted with permission from The Journal of Bone and Joint Surgery, Inc).

adherence of the olecranon cartilage to the trochlea. The triceps is elevated off the bone with a rasp of by finger dissection. All unstable hardware is removed. Nonunion tissue is debrided sharply. After clearing scar tissue and posterior capsule, the anterior capsule and scar can be released allowing increased joint mobilization (Fig. 5). If motion remains limited, the lateral collateral ligament can be osteotomized, allowing the joint to be hinged open on the medial ligamentous structures for further release.

\section{Fixation}

With a $2.0-\mathrm{mm}$ drill bit, the medullary canal is opened on both sides of the nonunion until blood is seen to egress from the canal. Realignment and stable reduction when anatomy is distorted can be facilitated by creating a trough in the distal fragment into which the shaft is impacted. Position of the distal segment in relation to the shaft should be carefully checked to assure restoration of the "carrying angle" in the anteroposterior plane and the flexion condyles in the lateral plane. Occasionally, a malunion of the condyles is present that impedes motion. Corrective osteotomy can realign the intra-articular component. All fragments are temporarily reduced with K-wires. The condylar block is reduced to the shaft with two crossed $\mathrm{K}$-wires. Definitive fixation is performed with plates. The changed anatomy will often preclude use of anatomic plates, thus requiring customized fixation. Locking plates nowadays allow better and more versatile fixation with $3.5-\mathrm{mm}$ screws along the shaft and metaphysis and 2.7-mm peri-articular screws (Fig. 6). To maximize fixation stability, we prefer placing two long screws from distal thru the plates crossing from medial to lateral and from lateral to medial. Sometimes, there is screw crowding precluding easy drilling. With the oscillating drill mode, the drill often will find its way. Subsequent cross threading of screws (intentional or non-intentional) will actually increase the holding power of the screws (Fig. 7). Tension band fixation can augment fixation in osteopenic bone as it relies on muscle and ligament attachment to bone and not so much on bone quality. Autologous bone graft is the gold standard for nonunion treatment. With a chisel, small bone cuts are made on both sides of the nonunion, leaving soft tissues attached as much as possible and bone graft is added. The olecranon osteotomy is fixed with a figure-of-eight tension band or tension band plates with an intramedullary screw. If the ulnar nerve had preoperative symptoms, anterior nerve transposition can be performed. The elbow is placed in a removable splint. Gentle-active and active-assisted range of motion exercise is immediately started under guidance of a physical or occupational therapist, allowing light functional activities as pain allows. Healing is generally seen at 35 months after index surgery. Once the nonunion is healed, the patient is allowed unrestricted activities.

\section{Results}

We evaluated 62 patients with a delayed or nonunion of the distal humerus. All patients were treated using the protocol
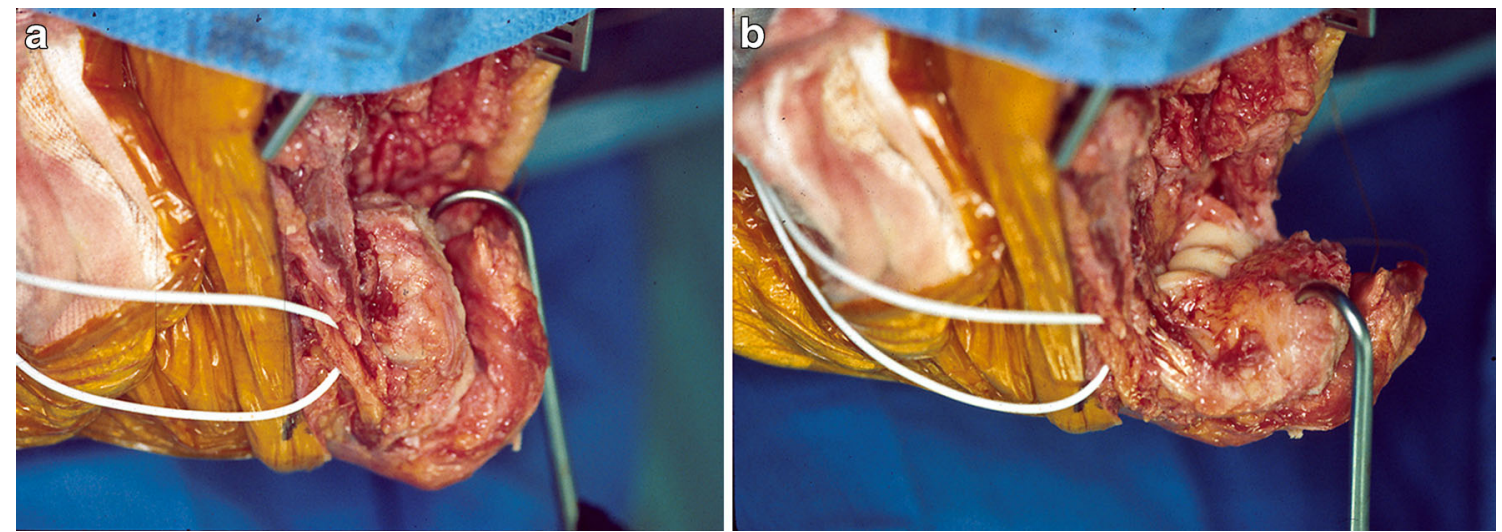

Fig. 5. Intraoperative photographs showing the amount of motion of the distal fragment after an extensile release. 

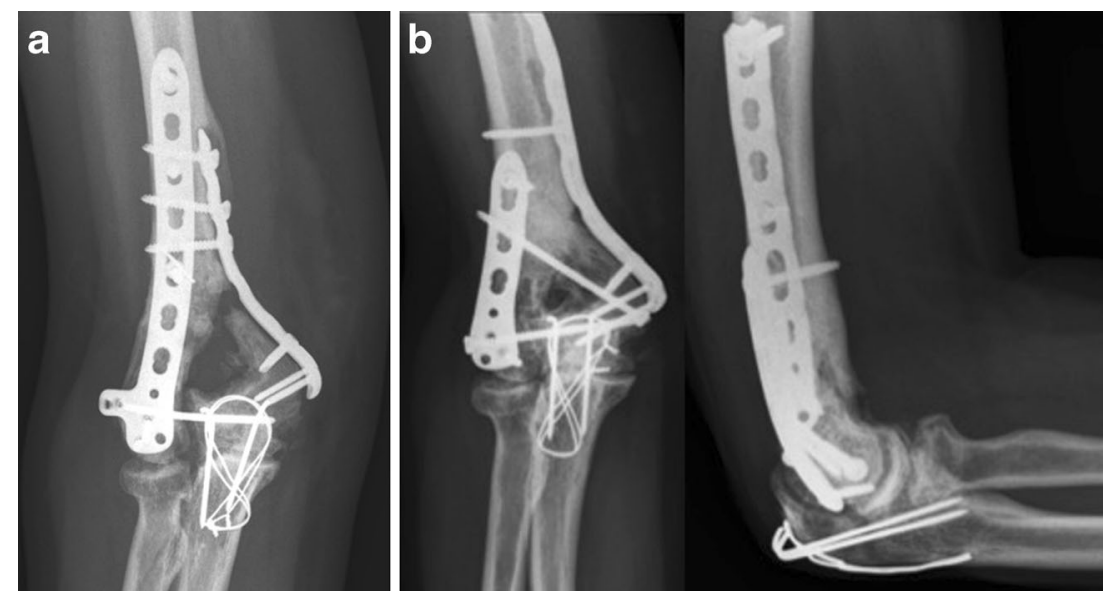

Fig. 6. Anatomic locking plates $(3.5 \mathrm{~mm}$ proximal and $2.7 \mathrm{~mm}$ distal) provide an increased number of fixation options. This patient had a nonunion of her distal humerus fracture that showed positive cultures for Enterobacter cloacae (a). Revision internal fixation with new plates bone graft and antibiotics resulted in healing as demonstrated on AP and lateral radiographs (from left to right); (b).

as outlined above. Over the years, fixation devices have been modified and improved. The concepts of debridement, alignment, rigid fixation, bone grafting, and early motion have remained current.

Since our initial series published in 2003, we have treated an additional 10 patients. To date, this remains the largest series in the literature. The combined patient cohort with follow-up at least until complete healing now includes 32 male and 30 females with an average age of 48 years (range 16-88). The average length of time between injury and index surgery was 21 months (range 2-204). Indications for referral were pain, loss of function, instability, or

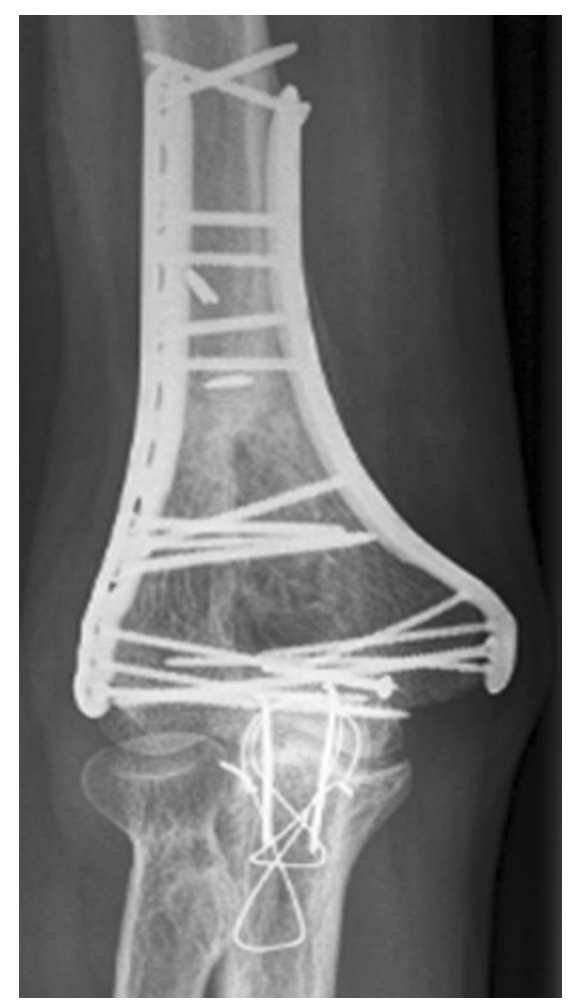

Fig. 7. Using parallel plating, there can be "crowding" of the screws distally. Cross threading of these screws might actually increase the holding power of the fixation. a combination. Thirty-one nonunions were supracondylar, 6 were transcondylar, 4 intercondylar, 13 T-type, 6 medial condylar, and 2 lateral condylar. A total of 47 patients had undergone previous internal fixation with an average number of previous operations of 1.5 (range 1-9). The operative approach used at the index procedure (leading to healing) was an olecranon osteotomy in 36 patients; in 26 , a triceps splitting, reflecting, or paratricipital approach was done. Six patients had an infection prior to our index surgery. None had an active infection at the time our index surgery. Nineteen had an isolated preoperative ulnar neuropathy and two had a radial nerve deficit. All patients were followed by the respective surgeons until healing.

All but one patient healed their nonunion. Average time to union was 6.8 months (range $2-45$ ). The average range of motion (ROM) at latest FU was $86^{\circ}$ (range 10-140). Complications included two superficial infections (successfully treated with antibiotics), two deep infection (for which irrigation and debridement and antibiotics), and new ulnar neuropathies in five patients. Two patients were found to have positive intraoperative cultures and were treated with 6 weeks antibiotics, which eradicated the infection. One patient developed a compartment syndrome caused by anasarca and recovered completely after fasciotomy. Another patient developed a radial and median neuropathy based on swelling in a radiated elbow with very constrictive soft tissue. He underwent emergent nerve releases. The median nerve recovered completely, but the radial nerve did not recover. Six patients $(10 \%)$ underwent additional surgery after healing. Four patients underwent hardware removal, one underwent hardware removal and removal of heterotopic bone, and one underwent ulnar neurolysis.

\section{Discussion}

A distal humeral nonunion often leads to a flail and painful arm. There are very few contraindications for surgical treatment. In the English literature, since the early 1980s, 24 publications have been published specifically on the 


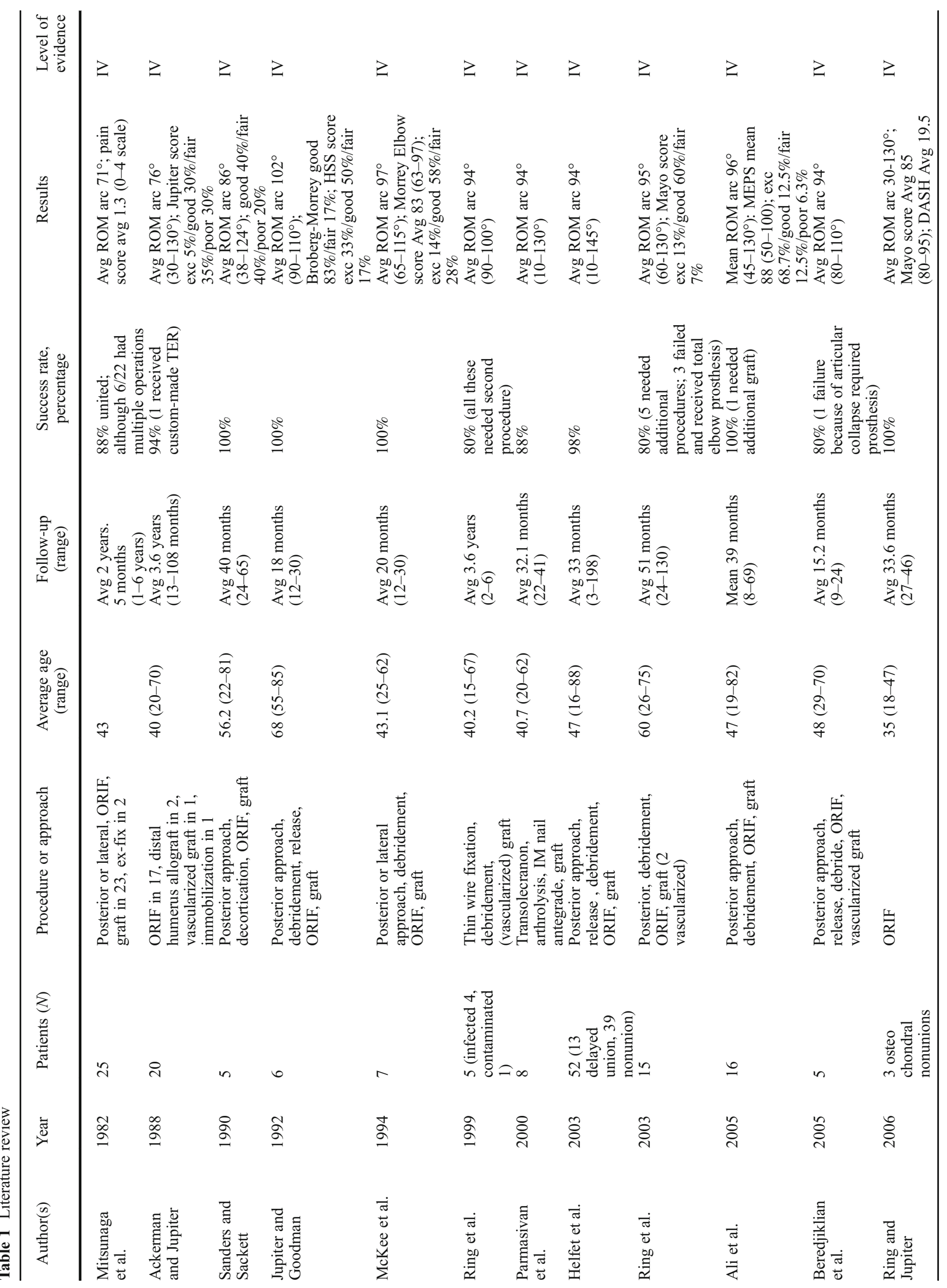




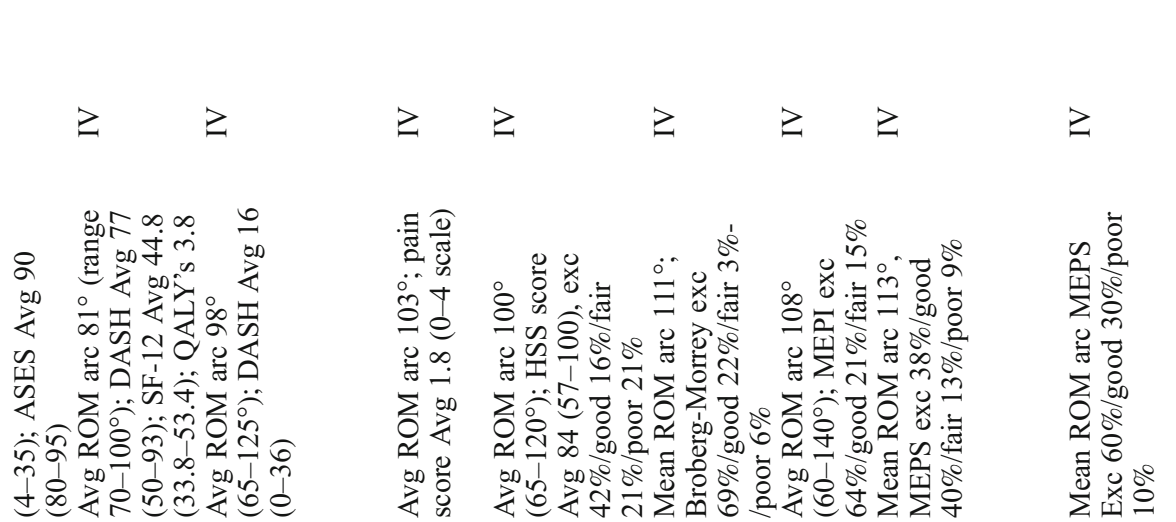

@̊

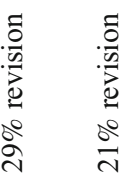

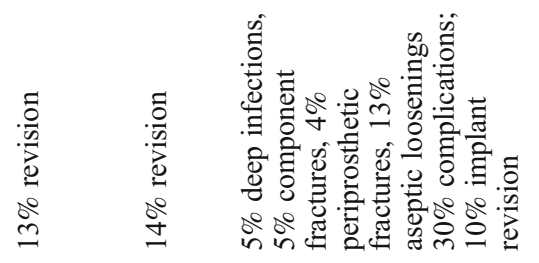

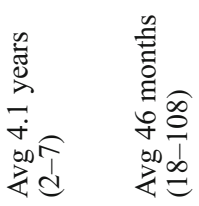

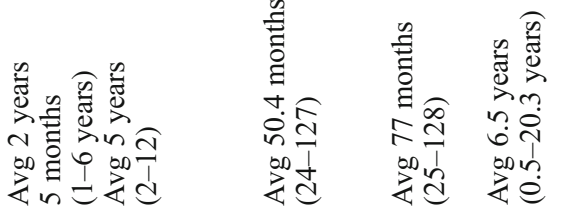

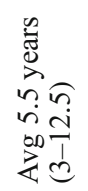

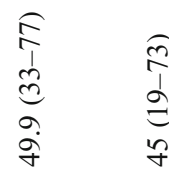

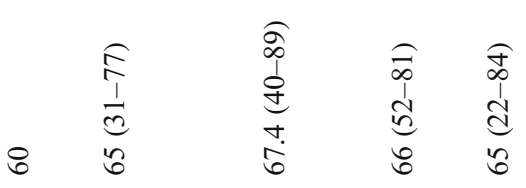

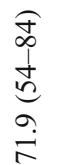

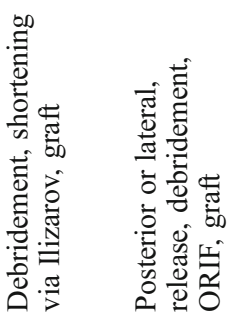

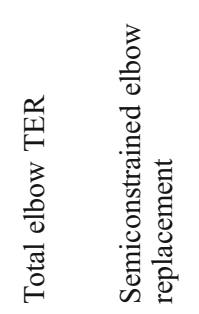

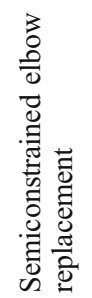
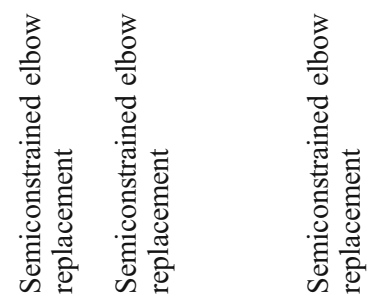

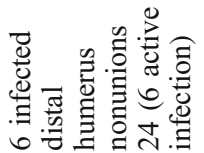

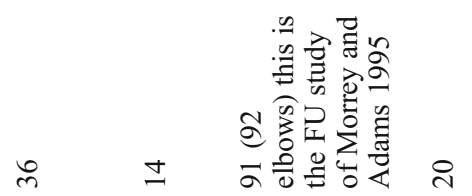

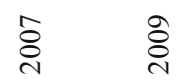

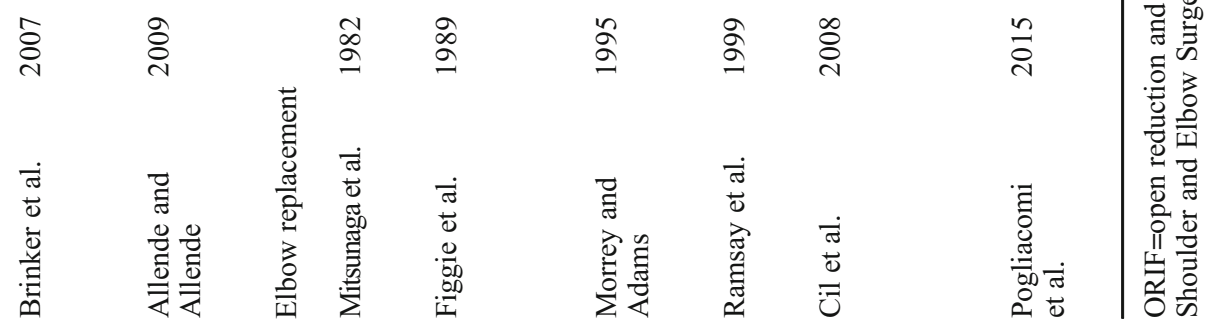

ڤ

ì 

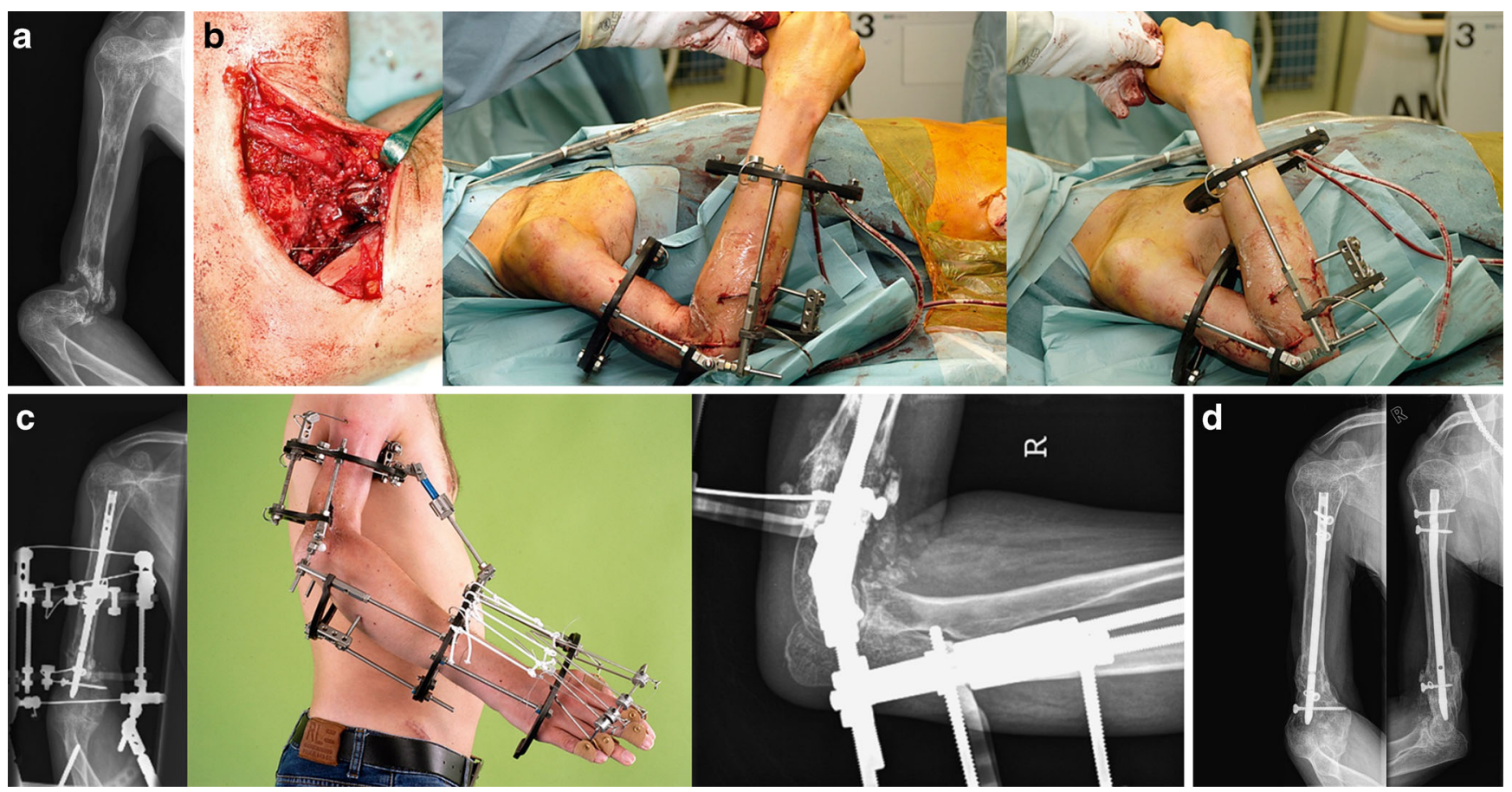

Fig. 8. The Ilizarov can be an extremely useful tool in complex cases not amenable to open reduction and internal fixation. A 26-year-old medical student presented with a distal humerus nonunion. As an 8-year-old, he underwent chemotherapy and radiation for an Ewing sarcoma of the humerus. At age 12, he sustained a distal humerus fracture treated with a cast for 2 years. Numerous surgeons were consulted during these years but surgical therapy was felt too risky as his upper arm had essentially remained the same size as when he was 8 years old with a thickened stiff skin and soft tissue cuff around the nonunion. He functioned reasonably well and entered medical school anticipating a career in plastic surgery. During his medical school, he developed increasing pain and instability of the arm and presented to us. Motion was limited to the nonunion site with a stiff elbow joint as seen on the lateral radiograph (a). Formal ORIF using an open approach was not an option. We referred the patient to an expert in Ilizarov techniques (Dr. Dror Paley) who agreed to operate in a combined procedure with the authors. Via a minimal approach the nonunion was debrided and an intramedullary nail was placed as an internal strut and an Ilizarov frame with an elbow hinge was then placed (b). Autologous bone graft was added locally. AP radiograph, clinical photo and lateral flexion radiograph (from left to right) illustrates final construct (c). In the next 24 hours, he developed increasing swelling and a median and radial nerve deficit (likely because of anasarca because of compromised lymph outflow). Exploration of the median and radial nerves was done on post-operative day two; additional bone graft was added at 6 months. At that time, the Ilizarov frame was removed and the nail was locked proximally. His nonunion healed as seen in AP and lateral radiographs (from left to right), (d). The median nerve fully returned; the radial nerve deficit remained complete. Eleven years later, he is pleased with the outcome - despite the radial nerve deficit. There is no pain and his elbow is stable. He is now working as a radiologist and has returned to all athletic activities including downhill skiing.

treatment of distal humeral nonunions [1-24]. Most series describe the results after internal fixation $[1-3,8-12,19$, $21,23]$, whereas two small subgroups used total elbow replacement $[6,7,13,15,17,18]$ or thin wire (Ilizarov) fixation $[5,20]$. A summary of the pertinent literature to date is given in Table 1 and illustrates that success rates of formal open reduction and internal fixation of a distal humeral nonunion are high with acceptable complications. It should be noted that all studies were level IV evidence. As this is a rather unusual clinical problem, it is very unlikely that prospective trials will ever be done. Union rate when treated with open reduction and internal or thin wire fixation in these studies ranged between 80 and $100 \%$ (average 93\%) with a total range of flexion-extension motion arc at latest follow-up reported between 71 and $102^{\circ}$ (average $91^{\circ}$ ).

Historically, arthrodesis of the elbow was considered an alternative for a flail, non-reconstructable joint. However, obtaining bone fusion is difficult when bone stock is limited.
As daily activities are limited with a fused elbow, few patients will choose this as salvage.

Distraction of the elbow joint interposing a layer of soft tissue (usually tensor fascia late autograft) was popularized by the Mayo Clinic. The technique is difficult with inconsistent outcomes. Especially, instability and weakness are a concern. Morrey reported on two patients who had their distal humerus nonunion treated by distraction arthroplasty [14]. Only one had a satisfactory result.

Urbaniak's series of 10 cadaveric elbow transplants describe 4 patients treated with an elbow allograft for a distal humeral nonunion that were followed for 1-6 years [24]. Indications were disabling elbow joint symptoms in patients who refuse an arthrodesis or are not a candidate for prosthetic replacement because of excessive bone loss or young age. This unique series had significant complications of degenerative joint changes resembling neuropathic joints, nonunion of the allograft-host junction, resorption of the allograft, and disease transmission. 
Given these concerns, it should be considered a salvage procedure.

Elbow replacement is a good salvage procedure in the low-demand, elderly (>70 years) patient $[6,7,12,15,17$, 18]. Its drawbacks are a limited lifespan, component fracture, loosening, and subsequent need for revision. Table 1 shows that the revision rates are high between 10 and $29 \%$. Total reported range of motion arc after total elbow replacement is higher, being an average of $107^{\circ}$ (range 100-113 than for those patients treated with internal fixation (average $91^{\circ}$, range $71-102^{\circ}$ ). Results of prosthetic elbow replacement as salvage after trauma are not as good or predictable when done as a primary procedure. It should be noted that heavy lifting is to be discouraged with a total elbow replacement and young active patients might not accept these limitations. Most studies to date come from a very experienced group at the Mayo Clinic and might not be easily reproduced by others $[6,15,18]$. Indications for replacement rather than internal fixation are (1) a distal humerus that is either too osteopenic or comminuted for reduction and fixation; and (2) extensive cartilage loss as seen in rheumatoid arthritis, posttraumatic arthritis, or ankylosis. The "ideal" candidate is the sensible older patient with good soft tissues and a retained osteopenic fragment with retention of muscular attachments and epicondyles as required for soft tissue balancing.

For infected distal humerus nonunions or those with compromised soft tissues, the Ilizarov technique of thin wire fixation is a good-but difficult-alternative [5, 20]. There is a steep learning curve and patients need to be motivated as the frame is cumbersome. We have limited experience with this technique (Fig. 8). Two small series using Ilizarov fixation for a distal humeral nonunion in the English literature have been published. The series of Jupiter et al. showed an $80 \%$ union rate in 5 patients but these patients all needed more than 1 procedure [20]. Brinker's series presented a single-stage procedure with debridement, release, shortening, Ilizarov frame fixation, and bone grafting. Using this protocol, they reported $100 \%$ healing in 6 patients [5]. It should be noted that these were very complex cases where formal internal fixation was not feasible because of soft tissue issues or active infection.

Presumed aseptic nonunions form a subgroup that might be underestimated. It is important always to consider infection as an underlying reason for nonunion, even if there are no obvious clinical signs of infection. Especially, Propionibacterium acnes is known to be associated with upper extremity nonunions. Always obtain deep cultures and customize treatment in collaboration with an infectious disease specialist.

In conclusion, internal fixation and bone grafting remains the treatment of choice for a nonunion of the distal humerus. Our results and those of others have shown careful preoperative planning, extensile approach, thorough debridement, and release of scarred soft tissues are essential to a successful reduction and optimal realignment. Rigid fixation with versatile locking plates and liberal use of bone graft will maximize the union rates and the reestablishment of a functional elbow in most patients.

\section{Compliance with Ethical Standards}

Conflict of Interest: Johanna C. E. Donders, MD; Dean G. Lorich, MD; David L. Helfet, MD; and Peter Kloen, MD, PhD, have declared that they have no conflict of interest.

Human/Animal Rights: All procedures followed were in accordance with the ethical standards of the responsible committee on human experimentation (institutional and national) and with the Helsinki Declaration of 1975, as revised in 2008 (5).

Informed Consent: Informed consent was waived from all patients for being included in the study. Additional consent was obtained from all patients for whom identifying information is included in this article.

Required Author Forms Disclosure forms provided by the authorsare available with the online version of this article.

Open Access This article is distributed under the terms of the Creative Commons Attribution 4.0 International License (http:// creativecommons.org/licenses/by/4.0/), which permits unrestricted use, distribution, and reproduction in any medium, provided you give appropriate credit to the original author(s) and the source, provide a link to the Creative Commons license, and indicate if changes were made.

\section{References}

1. Ackerman G, Jupiter JB. Non-union of fractures of the distal end of the humerus. J Bone Joint Surg (Am.). 1988;70:75-83.

2. Ali A, Douglas H, Stanley D. Revision surgery for nonunion after early failure of fixation of fractures of the distal humerus. J Bone Joint Surg (Br.). 2005;87:1107-10.

3. Allende C, Allende BT. Post-traumatic distal humerus non-union: Open reduction and internal fixation: long-term results. Int Orthop. 2009;33:1289-94.

4. Beredjiklian PK, Hotchkiss RN, Athanasian EA, Ramsey ML, Katz MA. Recalcitrant nonunion of the distal humerus: treatment with free vascularized bone grafting. Clin Orthop Relat Res. 2005;435:134-9.

5. Brinker MR, O'Connor DP, Crouch CC, Mehlhoff TL, Bennett JB. Ilizarov treatment of infected nonunions of the distal humerus after failure of internal fixation: an outcomes study. J Orthop Trauma. 2007;21:178-84.

6. Cil A, Veillette CJ, Sanchez-Sotelo J, Morrey BF. Linked elbow replacement: a salvage procedure for distal humeral nonunion. $\mathrm{J}$ Bone Joint Surg (Am.). 2008;90:1939-50.

7. Figgie MP, Inglis AE, Mow CS, Figgie HE, III. Salvage of non-union of supracondylar fracture of the humerus by total elbow arthroplasty. J Bone Joint Surg (Am.). 1989;71:105865.

8. Gallay SH, McKee MD. Operative treatment of nonunions about the elbow. Clin Orthop Relat Res. 2000;370:87-101.

9. Helfet DL, Kloen P, Anand N, Rosen HS. Open reduction and internal fixation of delayed unions and nonunions of fractures of the distal part of the humerus. J Bone Joint Surg (Am.). 2003;85:33-40.

10. Jupiter JB. The management of nonunion and malunion of the distal humerus - a 30-year experience. J Orthop Trauma. 2008;22:742-50.

11. Jupiter JB, Goodman LJ. The management of complex distal humerus nonunion in the elderly by elbow capsulectomy, triple plating, and ulnar nerve neurolysis. J Shoulder Elbow Surg. 1992;1:37-46. 
12. McKee M, Jupiter J, Toh CL, Wilson L, Colton C, Karras KK. Reconstruction after malunion and nonunion of intra-articular fractures of the distal humerus. Methods and results in 13 adults. J Bone Joint Surg Br. 1994;76:614-21.

13. Mitsunaga MM, Bryan RS, Linscheid RL. Condylar nonunions of the elbow. J Trauma. 1982;22:787-91.

14. Morrey BF. Post-traumatic contracture of the elbow. Operative treatment, including distraction arthroplasty. J Bone Joint Surg (Am.). 1990;72:601-18.

15. Morrey BF, Adams RA. Semiconstrained elbow replacement for distal humeral nonunion. J Bone Joint Surg (Br.). 1995;77:67-72.

16. Paramasivan ON, Younge DA, Pant R. Treatment of nonunion around the olecranon fossa of the humerus by intramedullary locked nailing. J Bone Joint Surg (Br.). 2000;82:332-5.

17. Pogliocomi F, Aliani D, Cavaciocchi M, Corradi M, Ceccarelli F, Rotini R. Total elbow arthroplasty in distal humeral nonunion: clinical and radiographic evaluation after a minimum follow-up of three years. J Shoulder Elbow Surg. 2015;24:1998-2007.
18. Ramsey ML, Adams RA, Morrey BF. Instability of the elbow treated with semiconstrained total elbow arthroplasty. J Bone Joint Surg (Am.). 1999;81:38-47.

19. Ring D, Jupiter JB. Operative treatment of osteochondral nonunion of the distal humerus. J Orthop Trauma. 2006;20:56-9.

20. Ring D, Jupiter JB, Toh S. Salvage of contaminated fractures of the distal humerus with thin wire external fixation. Clin Orthop Relat Res. 1999;359:203-8.

21. Ring D, Gulotta L, Jupiter JB. Unstable nonunions of the distal part of the humerus. J Bone Joint Surg (Am.). 2003;85:1040-6.

22. Sanchez-Sotelo J. Distal humeral nonunion. Instr Course Lect. 2009;58:541-8.

23. Sanders RA, Sackett JR. Open reduction and internal fixation of delayed union and nonunion of the distal humerus. J Orthop Trauma. 1990;4:254-9.

24. Urbaniak JR, Black KE, Jr. Cadaveric elbow allografts. A sixyear experience. Clin Orthop Relat Res. 1985;197:131-40. 